
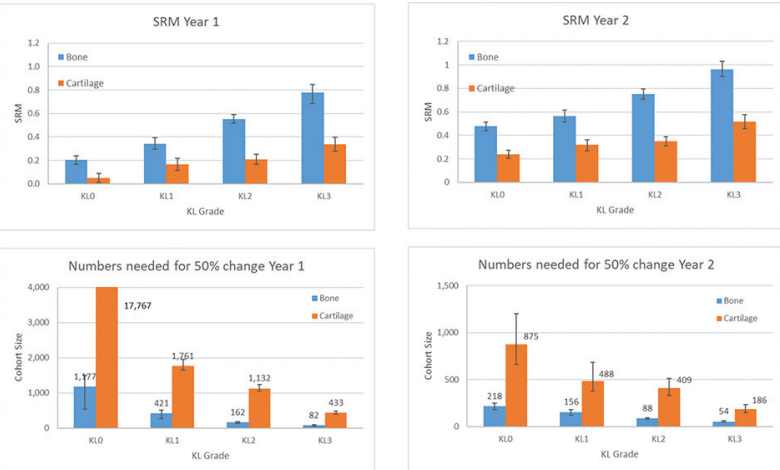

Abstract THU0425 - Figure 1. SRM and cohort numbers by KL grade for bone shape and cartilage thickness in 6,939 knees. Top row shows SRM values at 1 year (left) and 2 years for femur bone shape and central medial femur cartilage thickness, with $95 \%$ confidence limits calculated using a bootstrap method. Bottom row shows numbers needed per cohort arm, assuming $50 \%$ change, $80 \%$ probability, alpha 0.05 . Units are in vector units for bone shape, and $\mathrm{mm}$ for cartilage thickness.

Abstract THU0425 - Table 1. Responsiveness of bone shape and cartilage thickness by KL Grade

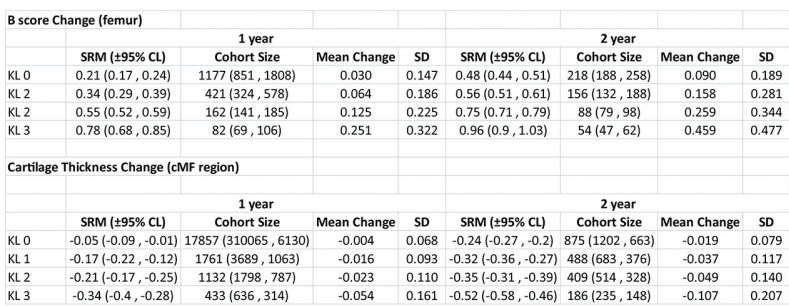

Detailed breakdown of SRM values, cohort size, mean change, and SD of that change for Figure 1. Units are in vector units for bone shape, and $\mathrm{mm}$ for cartilage thickness.

Disclosure of Interests: Michael Bowes Shareholder of: Stryker Corporation, Employee of: Stryker Corporation, Alan Brett Shareholder of: Stryker Corp, Employee of: Stryker Corp, Philip G Conaghan Consultant for: Flexion Therapeutics, AbbVie, Medivir, Merck Serono, Novartis, GlaxoSmithKline

DOI: 10.1136/annrheumdis-2019-eular.6992

\section{THU0426 EFFECTS OF SODIUM SUCCINATE AND HYALURONIC ACID IN KNEE OSTEOARTHRITIS TREATMENT}

Oleksandr Burianov $^{1}$, Liudmyla Khimion ${ }^{2}$, Taras Omelchenko ${ }^{1}$, Halyna Havryliuk ${ }^{2}$. ${ }^{1}$ Bogomolets National Medical University, Traumatology and Orthopedic, Kyiv, Ukraine; ${ }^{2}$ Shupyk National Medical Academy of Postgraduate Education, Family Medicine, Kyiv, Ukraine

Background: At present hyaluronic acid $(\mathrm{HA})$ is rather widely used in treatment of patients with osteoarthritis (OA). HA normalizes the properties of the synovial fluid; has a protective effects; promotes the cartilage nutrition and so improves the signs of $\mathrm{OA}$ and function of the joints. Sodium succinate (the salt of the succinic acid) helps to normalize intracellular metabolism and tissue respiration in hypoxic conditions via mitochondrial mechanism of action; normalizes acidic - alkaline balance; takes part in $\mathrm{K}^{+}$and $\mathrm{Ca}^{2+}$ transportation and provides antioxidant defense, - so is a promising compound for cartilage treatment

Objectives: To investigate the clinical efficacy of combination of hyaluronate and sodium succinate in treatment of early $O A$ stages

Methods: The study included 126 patients with knee OA (stages I-II, Kelgren and Lawrence), mean age $(54.3 \pm 2.7)$ years, among them - 75 women $(60 \%)$ and 51 men - (40\%). All enrolled patients had OA exacerbation (without clinically evident synovitis) and received standard OA treatment (NSAIDs, exercises, orthopedic devices) for 15 days; Gr.1 patients (58) also got 5 intra-articular injections of $1.1 \%$ hyaluronic acid, stabilized with sodium succinate ( $2 \mathrm{ml}$, once a week); patients of $\mathrm{Gr} .2$ (68) in addition to standard treatment received 5 intra-articular injections of $1,1 \%$ solution of non-stabilized HA (2 ml, once a week). Clinical observation and evaluation of the results were performed at the beginning of the treatment, at $6^{\text {th }}, 12^{\text {th }}$ and $24^{\text {th }}$ week after the study beginning
Results: During the treatment period, patients in both groups showed the positive changes in clinical signs and symptoms of $O A$ which led to the lowering of the general WOMAC index (from (78.3 \pm 4.1 ) in $\mathrm{Gr}$. 1 and (75.4 \pm 3.8) in Gr. 2 at the beginning of the study to $(27.9 \pm 2.6)$ and (29 $8 \pm 1.9$ ) accordingly at week 12 ( $p<0,05$ for both groups). The VAS score in both groups indicated a significant pain reduction, but the stability and duration of the clinical effect in the groups was different. In patients of Gr.1, the pain syndrome continued to decrease after 12 weeks till $24^{\text {th }}$ week, whereas in Gr.2 after the treatment course there was no significant changes in further pain regression after $6^{\text {th }}$ week point The changes in Lisholm score were also significantly better in Gr.1 than in Gr. 2 (before treatment $(21.7 \pm 4.6)$ and $(22.6 \pm 5.3)$, at week 6 $(86.4 \pm 5.7)$ and $(71.3 \pm 4.8)$, at week $12-(87.6 \pm 6.2)$ and $(63.8 \pm$ $5.3)$, respectively, $p<0,05$ between groups at week $12^{\text {th }}$

Conclusion: Combination of the hyaluronic acid and sodium succinate biochemical and physiologic properties in early stages of knee OA (as intra-articular injections) allows to increase the treatment efficacy, achieve better pain control, and more stable remission comparing to use of the hyaluronic acid alone

Disclosure of Interests: None declared

DOI: 10.1136/annrheumdis-2019-eular.6120

\section{THU0427 \\ THE LIFETIME RISK OF KNEE AND HIP REPLACEMENT FOLLOWING A GP DIAGNOSIS OF OSTEOARTHRITIS: REAL WORLD COHORT DATA FROM CATALONIA INCLUDING 48,311 PARTICIPANTS WITH UP TO 9 YEARS OF FOLLOW-UP}

Edward Burn ${ }^{1}$, David W Murray ${ }^{2}$, Gillian A Hawker ${ }^{3,4}{ }^{5}$, Rafael Pinedo-Villanueva ${ }^{1}$, Daniel Prieto-Alhambra ${ }^{1,6} .{ }^{1}$ Centre for Statistics in Medicine, NDORMS, University of Oxford, Oxford, United Kingdom; ${ }^{2}$ NDORMS, University of Oxford, Oxford, United Kingdom; ${ }^{3}$ Women's College Hospital, Women's College Research Institute, Toronto, Canada; ${ }^{4}$ Institute for Clinical Evaluative Sciences, Toronto, Canada; ${ }^{5}$ Department of Medicine, University of Toronto, Toronto, Canada; ${ }^{6}$ GREMPAL Research Group, Idiap Jordi Gol and CIBERFes, Universitat Autonoma de Barcelona and Instituto de Salud Carlos III, Barcelona, Spain

Background: The lifetime risk of knee and hip replacement following a primary care diagnosis of knee or hip osteoarthritis is unknown and overly negative beliefs about prognosis act as a barrier to implementing recommended management strategies.

Objectives: To estimate lifetime risk of knee and hip replacement follow ing a GP diagnosis of osteoarthritis and assess how this risk varies with patient characteristics.

Methods: Primary care and linked hospital data from Catalonia, covering 2006 to 2015, were used. Study participants had a newly recorded GP diagnosis of knee or hip osteoarthritis. Parametric survival models were specified for risk of knee/hip replacement and death following diagnosis. Survival models were combined and extrapolated using a Markov model and lifetime risk estimated for the average patient profile. The effects of age at diagnosis, sex, comorbidities, socioeconomic status, body mass index (BMI), and smoking on risk were assessed.

Results: 48,311 individuals diagnosed with knee osteoarthritis were included with a median follow-up of 4.3 years (IQR: 2.1 to 6.5 ) and of whom 2,561 underwent knee replacement. Respective figures for hip osteoarthritis were 15,105 individuals diagnosed with a median follow-up of 3.8 years (IQR: 1.8 to 6.1 ) and 1,247 hip replacements. The average participant's lifetime risk for knee replacement was $30 \%(95 \% \mathrm{Cl}: 25 \%$ to $36 \%)$ and for hip replacement was $14 \%$ (10\% to $19 \%)$. Notable patient characteristics influencing lifetime risk were age at diagnosis for knee and hip replacement, sex for hip replacement, and BMI for knee replacement. BMI increasing from 25 to 35 was associated with lifetime risk of knee replacement increasing from $24 \%(20 \%$ to $28 \%)$ to $32 \%(26 \%$ to $37 \%)$ for otherwise average patients.

Conclusion: Knee and hip replacement are not bound to happen for most after a GP diagnosis of osteoarthritis, with average lifetime risks of less than a third and a sixth, respectively. Patient characteristics influence lifetime risks with, most notably higher BMl associated with a meaningfully increased risk of knee replacement.

Disclosure of Interests: : Edward Burn: None declared, David W Murray Grant/research support from: Grants from Zimmer Biomet, Consultant for: Personal fees from Zimmer Biomet, Gillian A Hawker: None declared Rafael Pinedo-Villanueva: None declared, Daniel Prieto-Alhambra Grant/ research support from: Grants from Amgen, UCB Biopharma and Servier outside the submitted work, Consultant for: UCB Biopharma, Speakers bureau: Amgen

DOI: 10.1136/annrheumdis-2019-eular.5401 


\section{THU0428 THE EFFECTS OF NEUROMUSCULAR ELECTRICAL STIMULATION ON STRENGHT, PAIN, AND FUNCTION IN INDIVIDUALS WITH KNEE OSTEOARTHRITIS: A SYSTEMATIC REVIEW WITH META-ANALYSIS}

Camila Cadena ${ }^{1,2}$, Vinícius Bispo ${ }^{3}$, Karenina Modesto ${ }^{1}$, Gerson Cipriano ${ }^{4}$, João Luiz Durigan ${ }^{4}{ }^{1}$ Universidade de Brasília, Faculdade de Educação Física, Brasília, Brazil; ${ }^{2}$ Tribunal Superior Eleitoral, Fisioterapia, Brasília, Brazil; ${ }^{3}$ CEFITE, Brasília, Brazil; ${ }^{4}$ Universidade de Brasília, Fisioterapia, Brasília, Brazil

Background: Even thought Neuromuscular electrical stimulation (NMES) has been widely used as a non-pharmacological intervention in patients with knee osteoarthritis $(\mathrm{OA})$ to improve muscle strength and function, prior research has shown conflicting results regarding NMES effectiveness in knee OA treatment.

Objectives: The aim of this systematic review and meta-analysis was to investigate the effectiveness of NMES in muscle strengthen, pain, and function in individuals with knee $\mathrm{OA}$.

Methods: Only randomized controlled trials (RCTs) were included in this study. Two authors independently performed the study selection. We use Pubmed, Embase, LILACS, PEDro and Cochrane Central Register of Controlled Trials as data sources. The main outcome evaluated was muscle strength. Function and pain were assessed as secondary outcomes using the Western Ontario Macmaster (WOMAC) questionnaire and Timed get up and go (TUG) test. The methodological quality was assessed using PEDro scale.

Results: A total of 23,215 were initially identified. After selection of titles and abstracts, studies were selected for the full-text analysis, ten studies were included with a total sample size of 622 patients. The methodological quality of the selected studies was moderate, with a mean score of 5.5 on a $0-10$ scale (PEDro). The following analysis were performed:

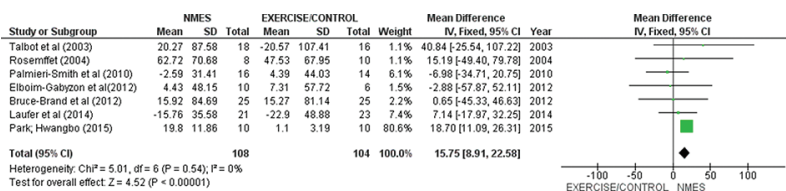

Abstract THU0428 - Figure 1. Meta-analysis of the effect of NMES on the increase of isometric muscle strength.

Legend: All measurements were standardized in $\mathrm{N} / \mathrm{m}$. A homogeneity in the data $\left(\mathrm{Chi}^{2}=5.01 ; \mathrm{I}^{2}=0 \%\right)$ was identified, with a significant difference in favor of NMES $(Z=4.52, p=0.01)$. NMES - Neuromuscular electro stimulation.

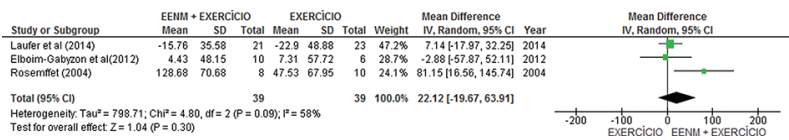

Abstract THU0428 - Figure 2. Meta-analysis of the effect of NMES associated with exercise vs conventional exercise in increasing isometric muscle strength.

Legend: A heterogeneity in the data was identified $\left(\mathrm{Chi}^{2}=4.80, \mathrm{I}^{2}=58 \%\right)$, with no significant difference in favor of NMES $(Z=1.04, p=0.30)$.

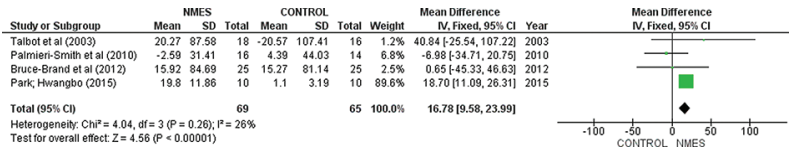

Abstract THU0428 - Figure 3. Meta-analysis of the effect of NMES vs control (No intervention) on isometric muscle strength

Legend: $A$ homogeneity in the data $\left(\mathrm{Chi}^{2}=4.04 ; \mathrm{I}^{2}=26 \%\right)$ was identified, with a significant difference in favor of NMES $(Z=4.56, p=0.01)$. $\mathrm{N}$

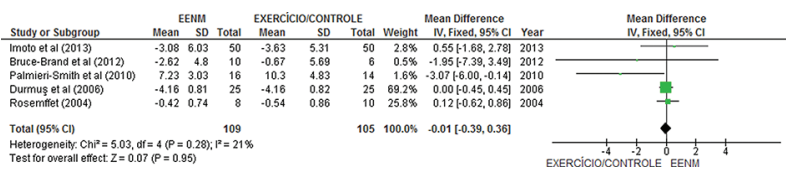

Abstract THU0428 - Figure 4. Meta-analysis of the effect of NMES on WOMAC pain
Legend: Data homogeneity $\left(\mathrm{Chi}^{2}=5.03 ; \mathrm{I}^{2}=21 \%\right)$ was identified, with no significant difference in favor of NMES $(Z=0.07, p=0.95)$. NMES Neuromuscular electro stimulation.

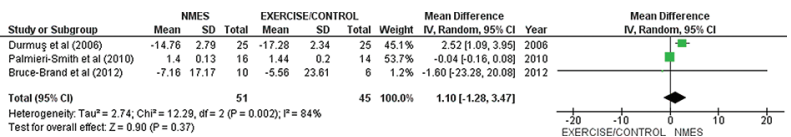

Abstract THU0428 - Figure 5. Meta-analysis of the effect of NMES on WOMAC function

Legend: Data homogeneity $\left(\mathrm{Chi}^{2}=12.29 ; \mathrm{I}^{2}=84 \%\right)$ was identified, with no significant difference in favor of NMES $(Z=0.90, p=0.37)$.

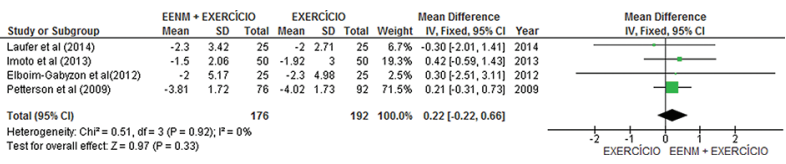

Abstract THU0428 - Figure 6. Meta-analysis of the effect of neuromuscular electrical stimulation on a secondary outcome: Timed Up and Go (TUG)

Legend: A homogeneity in the data $\left(\mathrm{Chi}^{2}=0.51 ; \mathrm{I}^{2}=0 \%\right)$ was identified with no significant difference in favor of NMES associated with exercise $(Z=0.97, p=0.33)$

Conclusion: NMES increased muscle strength in patients with OA compared to the active control group. However, the evidence to date did no demonstrate effects for pain control nor improvement in physical function.

\section{REFERENCES}

[1] Elboim-Gabyzon M, Rozen N, Laufer Y. 2013 Does neuromuscular electrical stimulation enhance the effectiveness of an exercise programme in subjects with knee osteoarthritis? A randomized controlled trial. Clinical Rehabilitation.27(3):246-257.

[2] De Oliveira Melo M, Aragão FA, Vaz MA. 2013 Neuromuscular electrical stimulation for muscle strengthening in elderly with knee osteoarthritis - A systematic review. Complementary Therapies in Clinical Practice.19(1):27-31

Acknowledgement: This research was supported by University of Brasília Disclosure of Interests: None declared

DOI: 10.1136/annrheumdis-2019-eular.3000

\section{THU0429 DISEASE BURDEN OF PERSONS WITH OSTEOARTHRITIS: RESULTS OF A CROSS-SECTIONAL ANALYSIS FROM A SURVEY LINKED TO CLAIMS DATA IN THE PROCLAIR PROJECT}

Johanna Callhoff ${ }^{1}$, Katinka Albrecht ${ }^{1}$, Imke Redeker ${ }^{2}$, Toni Lange ${ }^{3}$, Jens Goronzy ${ }^{3}$, Klaus-Peter Günther ${ }^{3}$, Angela Zink ${ }^{1,2}$, Jochen Schmitt ${ }^{4}$, Anne Postler ${ }^{3}$. ${ }^{1}$ German Rheumatism Research Centre, Epidemiology, Berlin, Germany; ${ }^{2}$ Charité University Medicine, Berlin, Germany, ${ }^{3}$ University Medicine Carl Gustav Carus Dresden, University Center of Orthopedics and Traumatology, Dresden, Germany; ${ }^{4}$ University Medicine Carl Gustav Carus Dresden, Dresden University Center for Evidence-Based Healthcare, Dresden, Germany

Background: Osteoarthritis $(\mathrm{OA})$ is a major reason for chronic pain, stiffness and functional limitation. While disease burden is frequently exam ined prior to surgery in knee and hip OA, burden in patients with unknown indication of joint surgery and the impact of polyarticular involvement are less often studied.

Objectives: The aim was to analyze factors associated with the burden of $\mathrm{OA}$, taking the pattern of joint involvement into account.

Methods: From a random sample of 8,995 persons with OA (ICD-10 codes M15 [polyarticular], M16 [hip] or M17 [knee]) from a large German statutory health insurance, 3,564 persons completed a questionnaire including the Western Ontario and McMaster Universities Osteoarthritis Index (WOMAC). Persons with knee, hip, concomitant knee and hip or polyarticular manifestation were compared concerning pain, stiffness, function, and impact on work/personal life, excluding asympomatic OA. Data were linked to dispensation records. The association of age, gender, BMI, symptom duration and WHO-5 with the WOMAC was assessed in multiple linear regression models.

Results: Persons with symptomatic knee $(1,130)$, hip (538), knee and hip (131) and polyarthritic (653) OA were included. Concomitant knee and hip OA was accompanied with the highest WOMAC values (mean 51), 